\title{
POTENCIAL DA ÁGUA NA FOLHA COMO UM INDICADOR DE DÉFICIT HÍDRICO EM MILHO'
}

\author{
JOÃO ITO BERGONCI ${ }^{2}$, HOMERO BERGAMASCH ${ }^{3}$, MOACIR ANTONIO BERLATO ${ }^{3}$ e ANTÔNIO ODAIR SANTOS ${ }^{4}$
}

\begin{abstract}
RESUMO - Este trabalho foi desenvolvido na Estação Experimental Agronômica da Universidade Federal do Rio Grande do Sul, localizada no município de Eldorado do Sul, nos anos agrícolas de 1993/94 e 1994/95. O objetivo foi avaliar o potencial da água na folha como indicador do déficit hídrico, em milho (Zea mays L.), relacionando-o ao potencial da água no solo. O experimento constou de três níveis de irrigação, desde a capacidade de campo até a ausência de irrigação. Os valores do potencial mínimo da água na folha foram desde -1,2 a -1,5 MPa em plantas irrigadas (na capacidade de campo) e de -1,6 a -2,0 MPa em plantas não irrigadas. O potencial mínimo da água na folha correlacionou-se com o potencial matricial da água no solo a $45 \mathrm{~cm}$ de profundidade $\left(\mathrm{r}^{2}=0,73\right)$, e mostrou ser um indicador adequado de déficit hídrico. O potencial da água na folha ao entardecer mostrou relação com o potencial mínimo da água na folha, indicando, assim, que pode ser utilizado como indicador de déficit hídrico. O potencial foliar de base apresentou diferenças evidentes entre os tratamentos extremos, mas não teve relação consistente com o potencial mínimo da água na folha.
\end{abstract}

Termos para indexação: Zea mays, relação planta-água, potencial hídrico do solo, índices de irrigação.

LEAF WATER POTENTIAL AS AN INDICATOR OF WATER DEFICIT IN MAIZE

ABSTRACT - This study was carried out at the Agronomic Experimental Station of the Federal University of Rio Grande do Sul, in Eldorado do Sul, RS, Brazil, during the agricultural seasons of $1993 / 94$ and 1994/95. The objective was to evaluate the leaf water potential as an indicator of the water deficit in maize (Zea mays L.), and its relation with the soil water potential. The experiment comprised three levels of irrigation, from field capacity to absence of irrigation. The values of the minimum leaf water potential ranged from -1.2 to $-1.5 \mathrm{MPa}$ in irrigated plants (field capacity) and from -1.6 to -2.0 MPa in nonirrigated plants. The minimum leaf water potential was well correlated to the matric water potential measured at $45 \mathrm{~cm}$ deep $\left(r^{2}=0.73\right)$. The sunset leaf water potential showed relationship with the minimum leaf water potential indicating it to be an indicator of water deficit. The predawn leaf water potential showed clear differences between the utmost treatments, but did not show any consistent relationship with the minimum leaf water potential.

Index terms: Zea mays, plant water relations, soil water potential, irrigation rates.

${ }^{1}$ Aceito para publicação em 7 de dezembro de 1999. Extraído da tese de doutorado do primeiro autor, apresentada à Faculdade de Agronomia da Universidade Federal do Rio Grande do Sul (UFRGS).

${ }^{2}$ Biólogo, Dr., Prof. Adjunto, Dep. de Botânica, UFRGS, Rua Paulo Gama, s/n, CEP 90043-900 Porto Alegre, RS. E-mail: joao.bergonci@ufrgs.br

${ }^{3}$ Eng. Agrôn., Dr., Prof. Adjunto, Faculdade de Agronomia, UFRGS, Av. Bento Gonçalves, 7712, CEP 91540-000 Porto Alegre, RS. Bolsista do CNPq. E-mail: homerobe@vortex.ufrgs.br, mberlato@ea-poa.com.br

${ }^{4}$ Eng. Agrôn., M.Sc., Seção de Climatologia Agrícola, Instituto Agronômico (IAC), Caixa Postal 28, CEP 13001-970 Campinas, SP. E-mail: odairsan@barao.iac.br

\section{INTRODUÇÃO}

O milho é uma cultura de grande interesse para o Estado do Rio Grande do Sul; sua demanda, nesse Estado, é crescente, mormente em face da expansão das atividades ligadas à criação de animais na região. Seu rendimento apresenta elevada variabilidade entre safras, causada pelas variações da precipitação pluvial. Diante desses aspectos, torna-se importante estudar possíveis indicadores de déficit hídrico referentes a essa cultura. O potencial de água da folha descreve o estado energético dela, cujos gradientes explicam os fluxos da água no sistema 
solo-planta-atmosfera. Embora haja variação ao longo do dia, mesmo em plantas irrigadas, esse parâmetro descreve o estado hídrico da planta, e tem sido muito utilizado em estudos das relações hídricas dos vegetais (Hsiao, 1973; Sionit \& Kramer,1976; Westgate, 1994). O valor do potencial da água indica a diferença entre o estado energético dela no sistema considerado e num estado de referência. No estado de referência, o potencial da água é considerado zero.

As medições do potencial da água na folha requerem alguns cuidados, a fim de que os dados obtidos reflitam o verdadeiro estado hídrico da planta. No caso do uso da câmara de Schollander, a pressurização deve ser lenta e deve-se evitar a perda de água após a coleta da folha, colocando-a em saco de plástico. Além disso, o tempo entre a retirada da folha e a medição deve ser o menor possível. A determinação do ponto de equilíbrio, isto é, quando a seiva inicia a saída do xilema é, por vezes, difícil de ser feita, e depende do tipo de planta, o que pode induzir a erros (Bennett, 1990). A utilização de lupa e iluminação eficiente auxilia esta determinação, diminuindo a incidência de erros de leitura.

Visto que muitas plantas, entre as quais o milho, apresentam capacidade de ajuste osmótico em resposta ao déficit de água (Tanguilig et al., 1987; Premachandra et al., 1992), o potencial de pressão é, freqüentemente, um melhor indicador do estado hídrico da planta, uma vez que ele se relaciona mais diretamente com a expansão celular. O potencial de pressão pode ser obtido diretamente, empregandose a técnica "pressure probe," a qual mede apenas o potencial de uma célula; mas, é dispendiosa, e nem sempre disponível. Também pode ser obtido indiretamente, por meio de medições dos potenciais da água total e osmótico e de curvas de pressão-volume, que demandam muito tempo. Por essas razões, tem sido utilizado o potencial total da água como indicador do estado hídrico da planta. Na interpretação das medidas desse potencial três fatores são geralmente considerados: o suprimento de água pelo solo, a demanda evaporativa da atmosfera e os mecanismos de controle da planta (Elfving et al., 1972).

Nos trabalhos da década de 70 , o potencial da água foliar era considerado o melhor indicador do estado hídrico da planta (Hsiao, 1973). Boyer (1970) observou que, à medida que o potencial da água na folha decrescia, a expansão foliar era inibida antes e mais severamente do que a fotossíntese e a respiração da planta do milho, da soja e do girassol. Potenciais da água na planta menores do que - $0,4 \mathrm{MPa}$ afetavam significativamente a expansão foliar, embora a fotossíntese máxima ocorresse com potenciais foliares entre -0,6 a -0,8 MPa.

Para Pandey et al. (1984), a matéria seca total e a produção de sementes é o resultado de uma resposta integrada da cultura ao estresse, desde a emergência até a maturidade fisiológica. Desta forma, o somatório de todas as medições de potencial da água pode ser um melhor indicador de tolerância à seca do que medições individuais.

O potencial da água foliar de base - ou simplesmente potencial de base - corresponde à medição feita pela manhã, antes do sol nascer. Assim, essa medida deve ser tomada num horário em que o comportamento iso-hídrico (em que o potencial da água na planta permanece elevado) ou aniso-hídrico (em que diminui acentuadamente nas horas mais quentes do dia) da cultura ou planta ainda não se manifestou. Desta forma, uma das grandes vantagens do potencial de base, quando comparado com outras variáveis que descrevem o estado hídrico da planta, é sua independência com relação às condições ambientais diurnas. Na realidade, essa variável reflete o equilíbrio de potencial entre a planta e o solo (Katerji \& Hallaire, 1984).

As medidas do potencial de base podem refletir a condição hídrica tanto do solo quanto da raiz, e são úteis para descrever o papel do estado hídrico da raiz e sua condutância hídrica em controlar o nível de perda de água pelas folhas (Reich \& Hinckley, 1989). $\mathrm{O}$ valor do potencial de base depende do estado hídrico do solo e da densidade e profundidade do sistema radicular. Katerji et al. (1988) observaram que a condutância estomática diminui sensivelmente quando é atingido um potencial de base crítico, que foi -0,4 MPa no tomateiro. Para Ansley et al. (1992), a relação entre condutância estomática, e potencial de base sugere que a água perdida através dos estômatos durante o dia depende, primeiramente, da reidratação noturna dos tecidos vegetais. Por outro lado, esses autores não encontraram uma relação clara entre o potencial da água foliar médio ao longo do dia e a condutância estomática. Para Reich \& 
Hinckley (1989), o controle estomático, ao amanhecer e ao entardecer, não é inerente à própria folha, mas depende de todos os processos envolvidos na dinâmica da água no sistema solo-planta.

Considerando que o potencial de base é medido antes da abertura estomática, parece haver um equilíbrio entre o estado hídrico da planta e o do solo. Embora exista uma relação entre o potencial de base e o potencial da água no solo, esta nem sempre é evidente. Dwyer \& Stewart (1984) observaram a ocorrência de uma relação linear entre o conteúdo de água no solo e o potencial de base entre 0 e $-0,4 \mathrm{MPa}$. Mas, a partir deste último valor, essa relação não se mostrou linear em milho, observando-se que a planta perde pouca água para o ambiente, ou seja, numa taxa menor do que $2 \mathrm{~mm} \mathrm{dia}{ }^{-1}$, indicando uma perda muito pequena de água pelo solo. Outros autores observaram que o potencial de base é relativamente insensível a mudanças no conteúdo de água no solo até que ele diminua em $22 \%$, a partir do que, a resposta é rápida (Ansley et al., 1992). A partir desse ponto, o potencial de base é muito sensível a mudanças em solos com diferentes potenciais matriciais.

Este trabalho teve como objetivo avaliar o potencial de água na folha do milho como indicador do déficit hídrico, relacionando-o com o potencial da água no solo.

\section{MATERIAL E MÉTODOS}

Foi realizado um experimento de campo nos anos agrícolas de 1993/94 e 1994/95, na Estação Experimental Agronômica da Universidade Federal do Rio Grande do Sul (EEA/UFRGS), situada no município de Eldorado do Sul, $\mathrm{RS},\left(30^{\circ} 6^{\prime} \mathrm{S}, 51^{\circ} 39^{\prime} \mathrm{W}\right.$, altitude $\left.46 \mathrm{~m}\right)$. O clima da Estação é do tipo Cfa, de acordo com a classificação de Köeppen, isto é, subtropical úmido de verão quente (Bergamaschi \& Guadagnin, 1990).

O solo é um Podzólico Vermelho-Amarelo, com relevo plano a levemente ondulado, textura franca no horizonte A e gradiente textural gradual ao longo do perfil (Rojas, 1998). O teor de argila nesse horizonte é próximo de $20 \%$, com cerca de $1,5 \%$ de matéria orgânica. Na camada de 0 a $30 \mathrm{~cm}$ de profundidade, o solo apresenta médias de umidades em base volumétrica de $0,22,0,20$ e 0,15 para potenciais matriciais de $-0,01,-0,033$ e $-1,5 \mathrm{MPa}$, respectivamente (Cunha, 1991).

O delineamento experimental foi em faixas, com cinco repetições, considerando que o sistema de aspersão em linhas utilizado não permite casualização do fator água (Hanks et al., 1980). A área ocupada pelo experimento tinha dimensões de 80 × $60 \mathrm{~m}$, no centro da qual foi instalado um lisímetro de pesagem de $5,1 \mathrm{~m}^{2}$ e resolução de $0,1 \mathrm{~mm}$ e com $90 \mathrm{~cm}$ de profundidade. Cada bloco era constituído por cinco parcelas de $16 \mathrm{~m}$ de comprimento por $3 \mathrm{~m}$ de largura, comportando, desta forma, quatro fileiras de milho.

Foram utilizadas sementes de milho, híbrido Pionner 3230, nos dois anos, semeadas em linhas, de orientação leste-oeste. O milho foi semeado no dia 28 de outubro de 1993 e no dia 4 de novembro de 1994. As sementes foram estabelecidas a uma profundidade de 5 a $7 \mathrm{~cm}$, com espaçamento de $75 \mathrm{~cm}$ entre linhas e $20 \mathrm{~cm}$ na linha, o que corresponde a 66.600 plantas por hectare.

A adubação foi efetuada nos dias 8 de outubro de 1993 e 14 de outubro de 1994, que constou de: $150 \mathrm{~kg} \mathrm{ha}^{-1} \mathrm{de}$ $\mathrm{P}_{2} \mathrm{O}_{5}$, na forma de superfosfato triplo; $120 \mathrm{~kg} \mathrm{ha}^{-1} \mathrm{de} \mathrm{K}_{2} \mathrm{O}$, na forma de cloreto de potássio e $40 \mathrm{~kg} \mathrm{ha}^{-1} \mathrm{de} \mathrm{N}$, na forma de uréia. Posteriormente, quando as plantas apresentavam entre 40 e $50 \mathrm{~cm}$ e no emborrachamento, foram incorporados na linha, $40 \mathrm{~kg} \mathrm{ha}^{-1}$ de N, também sob a forma de uréia.

O sistema de irrigação utilizado foi por aspersão em linha, constituído de uma linha de 12 aspersores (marca Perrot) de média pressão, colocados na direção longitudinal (leste-oeste) a $3 \mathrm{~m}$ de altura e com espaçamento de $6 \mathrm{~m}$ entre os mesmos, conforme metodologia descrita por Hanks et al. (1976). Para cada lado da linha de aspersores foram demarcados os tratamentos, cada um correspondendo a quatro filas de plantas (3 m de largura). Em 1993/94, os tratamentos aplicados foram: I4 com solo mantido próximo à capacidade de campo, monitorado através de tensiômetros e peso do lisímetro, sendo a irrigação efetuada quando os tensiômetros instalados a $45 \mathrm{~cm}$ de profundidade registravam o potencial matricial de $-0,05 \mathrm{MPa}$ e suspensa quando o peso do lisímetro atingia o valor correspondente ao solo drenado; $\mathrm{I} 2 \mathrm{com}$ dose de rega àquela correspondente a 77\% em relação a I4; e I0 sem irrigação. Em 1994/95, no tratamento I4, o solo foi mantido próximo à capacidade de campo, conforme critério adotado no ano anterior. $\mathrm{O}$ tratamento $\mathrm{I} 2$ teve dose de rega igual àquela correspondente a $75 \%$ em relação a I4 e I0 sem irrigação. A verificação da quantidade de água aplicada, em cada nível, foi feita fora do período experimental, através de um conjunto de potes coletores de $162,86 \mathrm{~cm}^{2}$ de superfície colocados nas duas entrelinhas centrais de cada parcela, na superfície do solo. A fim de quantificar a água aplicada, marcou-se o tempo de irrigação no momento da aplicação, sendo a aferição feita logo após a colheita, a fim de evitar erros decorrentes do fato de plantas atuarem como anteparo. Por pesagem do lisímetro, antes e depois de cada rega, 
foi controlada a dose de água aplicada no tratamento I4. Além disso, o lisímetro era pesado diariamente às $9 \mathrm{~h}$, quando eram feitas as leituras dos esquipamentos da estação meteorológica convencional localizada a $30 \mathrm{~m}$ da área experimental. Na Tabela 1 são apresentadas as quantidades de água aplicadas durante os dois períodos experimentais no tratamento correspondente à capacidade de campo.

O potencial da água na folha foi medido em câmara de pressão (Soil moisture), conforme descrito por Boyer (1967). Para cada tratamento foram feitas três repetições utilizando-se a folha imediatamente abaixo da espiga. As medições foram efetuadas quando as plantas já estavam pendoadas. As medições foram efetuadas das $6 \mathrm{~h}$ às $20 \mathrm{~h}$, com intervalo de duas horas entre elas. A média das medições efetuadas às $6 \mathrm{~h}$ foi considerada como potencial de base.

\section{RESULTADOS E DISCUSSÃO}

O potencial matricial do solo, nos dias em que foram feitas as medições do potencial da água na planta é apresentado na Tabela 2, onde se pode observar que os dias em que apresentou os menores valores foram 19 e 20 de janeiro de 1994 e 3 de fevereiro de 1994, indicando menor disponibilidade de água.

O potencial da água na folha medido em alguns dias, nos dois períodos experimentais, está representado nas Figs. 1 e 2. Nos dias em que a disponibilidade de água no solo foi baixa em plantas não

TABELA 1. Datas e quantidades de água aplicada correspondente ao tratamento em capacidade de campo, Eldorado do Sul, RS. 1993/94 e 1994/95.

\begin{tabular}{lclc}
\hline Data & $\begin{array}{c}\text { Irrigação } \\
(\mathrm{mm})\end{array}$ & Data & $\begin{array}{c}\text { Irrigação } \\
(\mathrm{mm})\end{array}$ \\
\hline $21 / 12 / 93$ & 15 & $16 / 12 / 94$ & 39 \\
$23 / 12 / 93$ & 17 & $23 / 12 / 94$ & 42 \\
$29 / 12 / 93$ & 9 & $29 / 12 / 94$ & 18 \\
$31 / 12 / 93$ & 15 & $6 / 1 / 95$ & 27 \\
$4 / 1 / 94$ & 26 & $23 / 1 / 95$ & 21 \\
$7 / 1 / 94$ & 20 & $1 / 2 / 95$ & 17 \\
$14 / 1 / 94$ & 30 & $9 / 2 / 95$ & 15 \\
$18 / 1 / 94$ & 12 & & - \\
$21 / 1 / 94$ & 19 & & - \\
$1 / 2 / 94$ & 41 & & - \\
\hline Total & 194 & & 179 \\
\hline
\end{tabular}

Pesq. agropec. bras., Brasília, v.35, n.8, p.1531-1540, ago. 2000 irrigadas (Tabela 2), o potencial da água na folha foi sensivelmente menor do que em plantas irrigadas, mostrando, assim, que pode ser indicador de déficit hídrico. Outro aspecto a ser considerado é o de que a recuperação do potencial da água nas plantas com déficit foi mais lento em resposta a uma baixa disponibilidade hídrica no solo (Tabela 1 e Figs. 1 e 2). Em outras palavras, a reposição da água, que fora perdida nos momentos de maior transpiração, foi mais rápida nas plantas com adequada disponibilidade hídrica, enquanto naquelas em que houve limitação essa reposição foi mais lenta.

Analisando a Fig. 3, observa-se uma relação quadrática entre o potencial mínimo da água na folha e o potencial da água na folha após o pôr-do-sol. Além disso, parece ocorrer um determinado valor de potencial mínimo (entre -1,2 e -1,5 $\mathrm{MPa}$ ) acima do qual ocorre a recuperação do potencial da água na folha após o fechamento estomático, valor este que poderia ser utilizado como critério indicador de déficit hídrico. Esses dados são confirmados pelas Figs. 1 e 2, onde se observa que, quando o potencial mínimo da água na folha for muito baixo, a sua recuperação ao entardecer não é satisfatória. Esses resultados parecem indicar que, havendo baixa disponibilidade de água no solo (Tabela 2), a recuperação do potencial da água na folha somente acontece após um período mais longo, à noite, a partir do fechamen-

TABELA 2. Potencial matricial do solo, medido com tensiômetro de coluna de mercúrio, a $45 \mathrm{~cm}$ de profundidade do solo submetido a diferentes lâminas de irrigação.

\begin{tabular}{lccc}
\hline \multirow{2}{*}{ Data } & \multicolumn{3}{c}{ Lâminas de irrigação $^{1}$} \\
\cline { 2 - 4 } & I0 & I2 & I4 \\
\hline & ----- Potencial matricial do solo (MPa) ---- \\
$19 / 1 / 94$ & $<-0,08$ & $-0,008$ & $-0,009$ \\
$20 / 1 / 94$ & $<-0,08$ & $-0,02$ & $-0,02$ \\
$26 / 1 / 94$ & $-0,057$ & $-0,05$ & $-0,02$ \\
$3 / 2 / 94$ & $-0,08$ & $-0,001$ & $-0,01$ \\
$19 / 1 / 95$ & $-0,006$ & $-0,04$ & $-0,008$ \\
$24 / 1 / 95$ & $-0,07$ & $-0,05$ & $-0,008$ \\
$30 / 1 / 95$ & $-0,04$ & $-0,03$ & $-0,005$ \\
$31 / 1 / 95$ & $-0,06$ & $-0,04$ & $-0,01$ \\
\hline 1 I0: sem irrigação; I2: dose de rega igual a 75\% de I4; I4: irrigação para \\
manter o solo próximo à capacidade de campo.
\end{tabular}


to estomático. Além disso, as curvas diárias de potencial da água na folha são praticamente simétricas em plantas com adequada disponibilidade hídrica, o que não ocorre nas submetidas a déficit hídrico, em face da dificuldade em repor a água perdida, causada pela sua baixa disponibilidade no solo. A mesma tendência foi observada por Slatyer (1967), Ehrler et al. (1978), Dwyer \& Stewart (1984) e Katerji et al. (1988) em diferentes culturas.
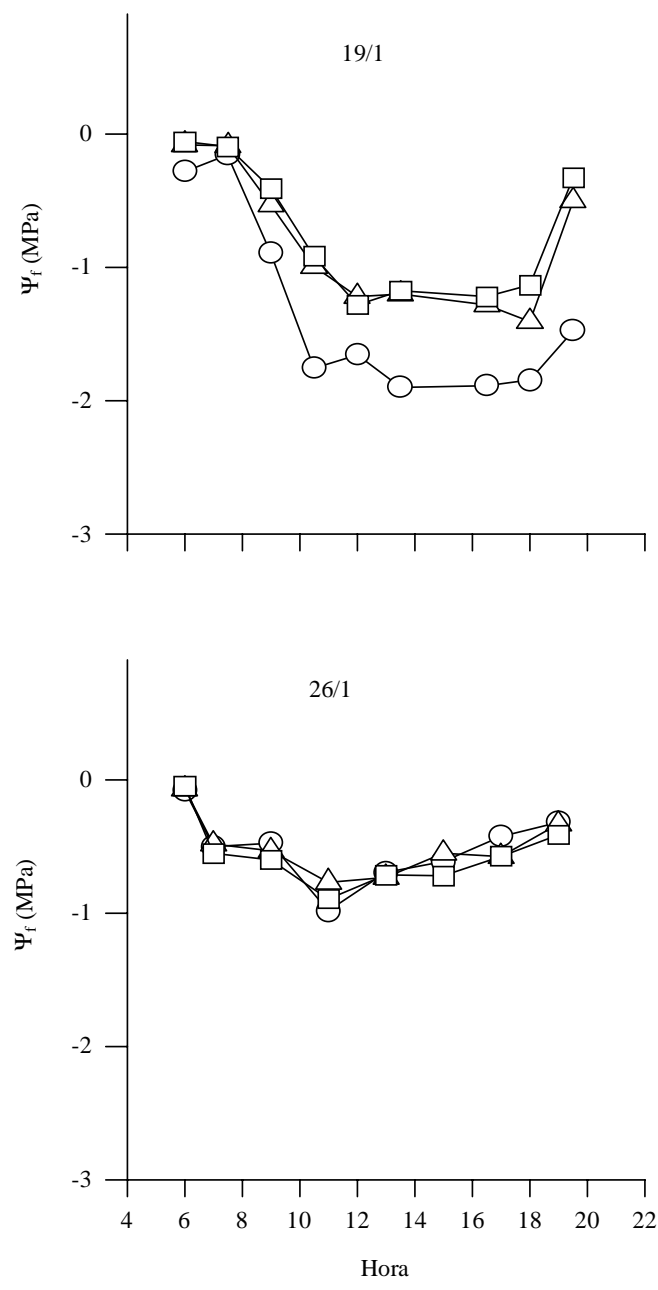

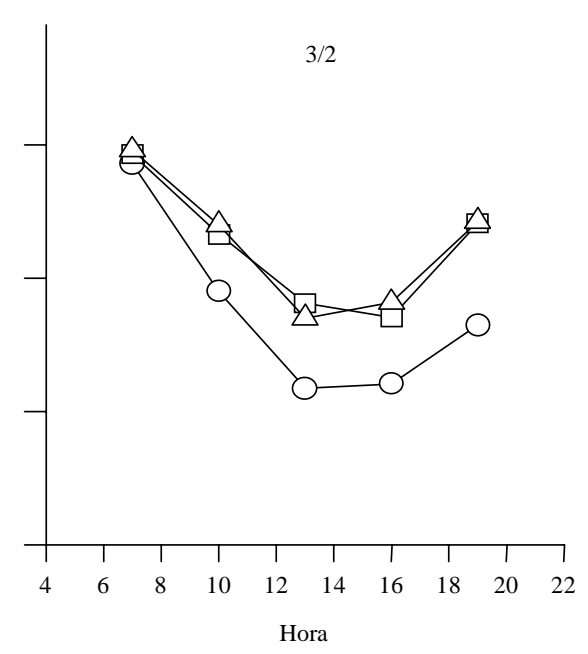

Um aspecto importante a ser considerado, quando se trata do fluxo de água do solo para as raízes, são as resistências implicadas no processo. Assim, um solo com baixo conteúdo de água terá sua resistência elevada, uma vez que sua condutividade hidráulica será baixa. Além disso, à medida que ocorre o secamento do solo, o contato entre este e a raiz decresce, dificultando a reposição de água na planta (Faiz \& Weatherly, 1978). Outro elemento que deve

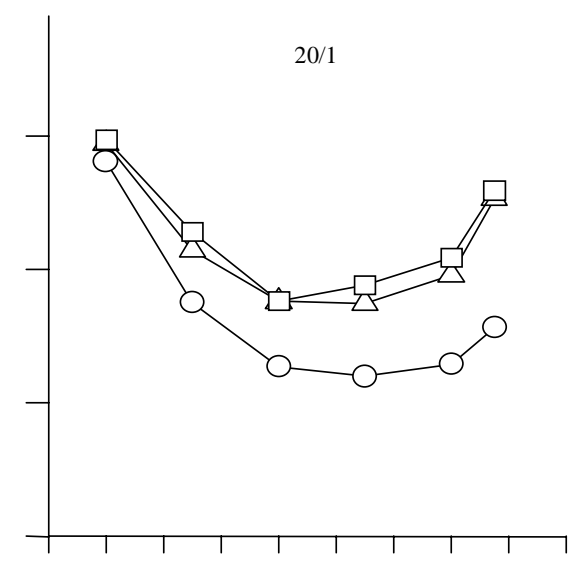

$-\square-\mathrm{I} 4$

FIG. 1. Potencial da água na folha $\left(\psi_{\mathrm{f}}\right)$ em diferentes horários nos dias 19/1, 20/1, 26/1 e 3/2 de 1994, em milho submetido aos tratamentos: sem irrigação (I0); dose de rega igual a $75 \%$ de I4 (I2) e irrigação para manter o solo próximo à capacidade de campo (I4). Eldorado do Sul, RS. 
ser levado em conta, quando se trata da reposição da água, é a resistência radicular, que, por vezes, é maior do que a própria resistência do solo (Reicosky \& Ritchie, 1976). Morizet \& Togola (1984) atribuíram
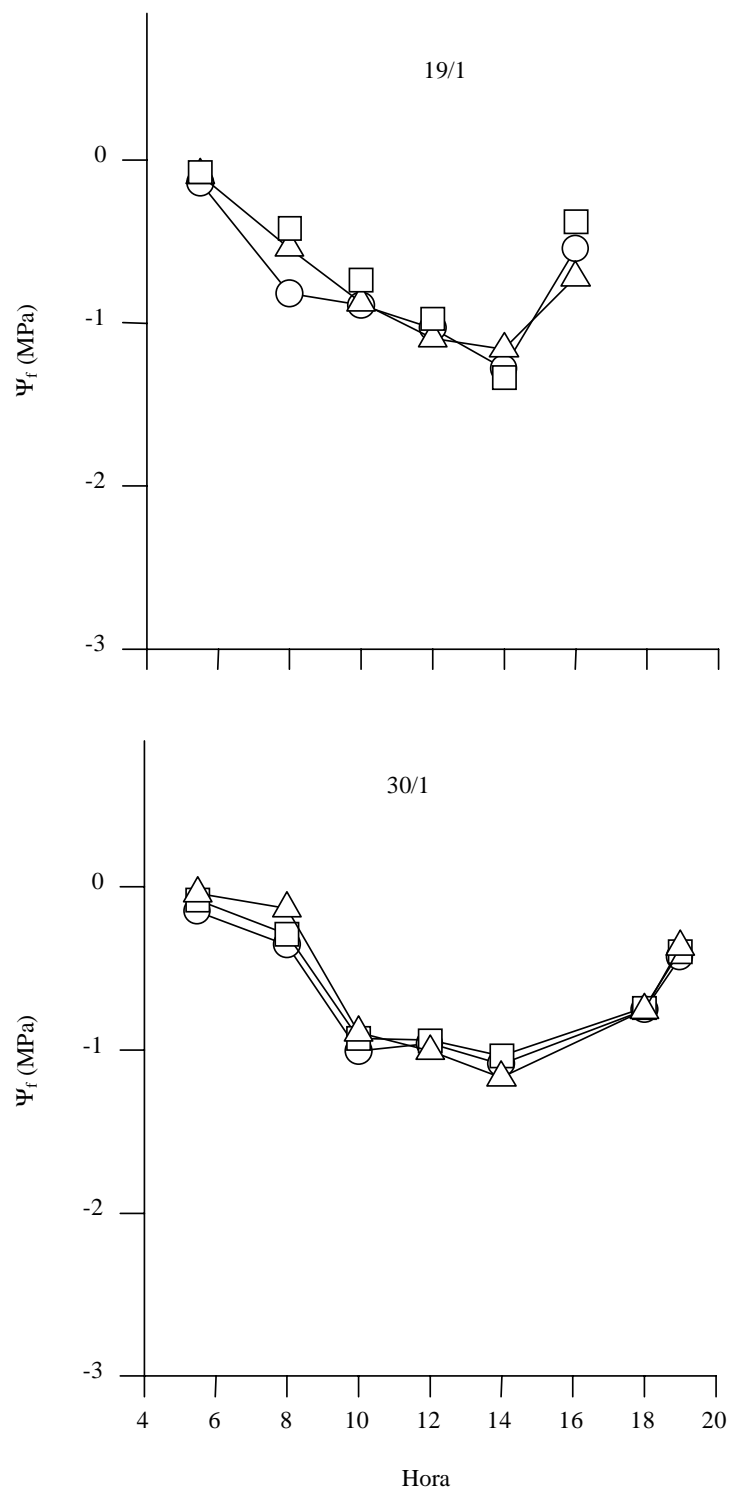

o menor consumo de água em milho em relação ao girassol, à sua maior resistência radicular.

Nos dias em que o déficit é inexistente ou pequeno (Tabela 2), o potencial da água na folha não apre-
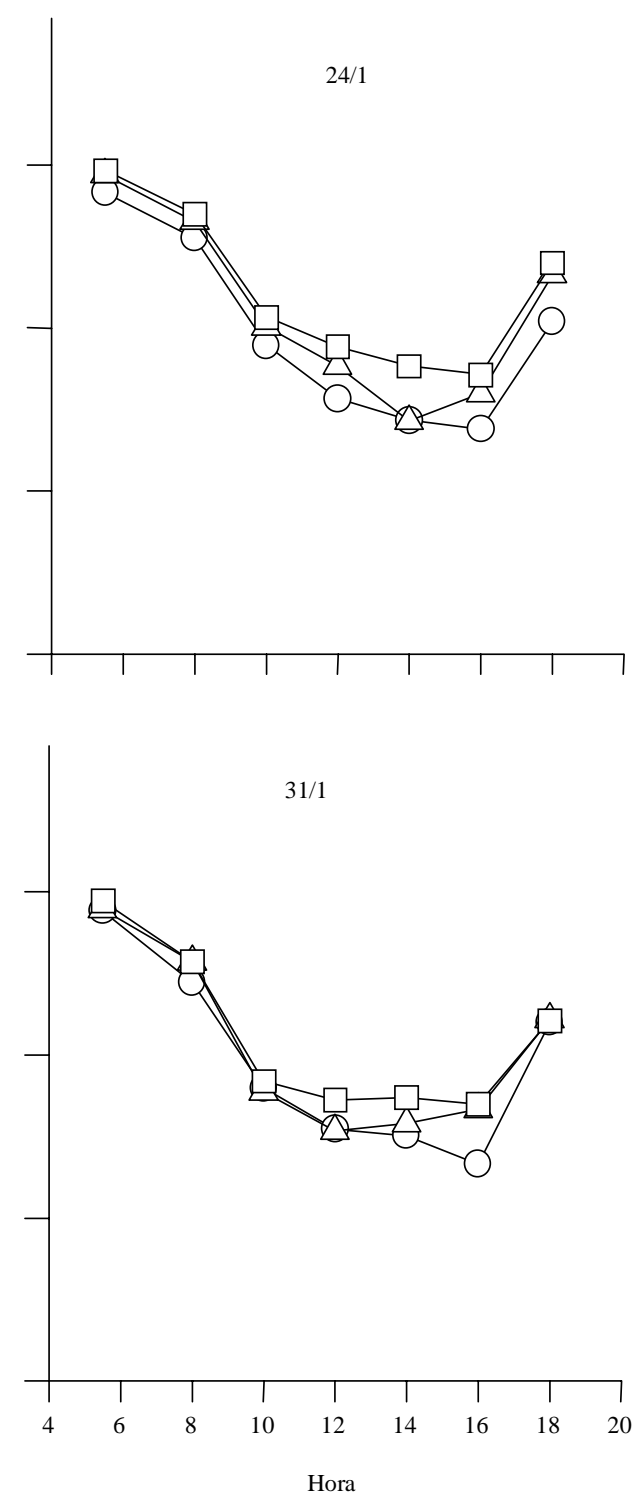

FIG. 2. Potencial da água na folha ( $\psi_{\mathrm{f}}$ ) em diferentes horários nos dias 19/1, 24/1, 30/1 e 31/1 de 1995, em milho submetido aos tratamentos: sem irrigação (I0); dose de rega igual a $75 \%$ de I4 (I2) e irrigação para manter o solo próximo à capacidade de campo (I4). Eldorado do Sul, RS. 
sentou diferenças entre tratamentos, e a recuperação do mesmo foi rápida, em conseqüência do suprimento adequado de água pelo solo (Figs. 1 e 2). Neste caso, o potencial da água na folha ao entardecer é praticamente o mesmo em plantas irrigadas e nas não irrigadas.

O potencial de base está representado na Fig. 4, onde se constatam diferenças entre os tratamentos, principalmente quando o déficit hídrico foi mais acentuado. Nos dias em que o déficit hídrico no solo foi pouco pronunciado, as diferenças são menores, indicando maior disponibilidade de água em todos os tratamentos (Tabela 2). No tratamento intermediário, o fato de o potencial de base não apresentar nítidas diferenças em relação ao irrigado poderia ser atribuído à maior profundidade do sistema radicular. Assim, em déficits não muito severos, as plantas com sistema radicular mais profundo exploram o solo em

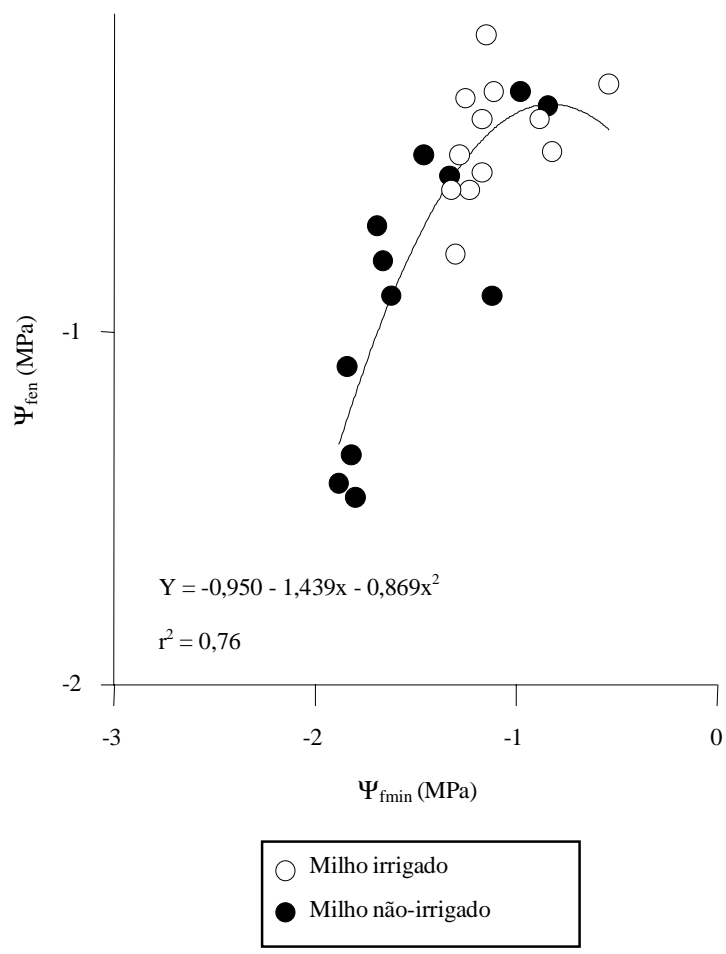

FIG. 3. Relação entre o potencial mínimo de água na folha $\left(\psi_{\text {fmin }}\right)$ e ao entardecer $\left(\psi_{\text {fen }}\right)$ em milho irrigado e não-irrigado nos períodos experimentais de 1993/94 e 1994/95. Eldorado do Sul, RS. locais que ainda apresentam água passível de ser absorvida (Sharp \& Davies, 1979; Morison \& Gifford, 1984). Crombie et al. (1988), efetuando estudos com espécies florestais, observaram uma estreita relação entre o potencial de base e a profundidade do sistema radicular. Outra possível causa poderia ser o fluxo ascendente de água no solo à noite, o qual seria suficiente para elevar o potencial de base. Resulta-
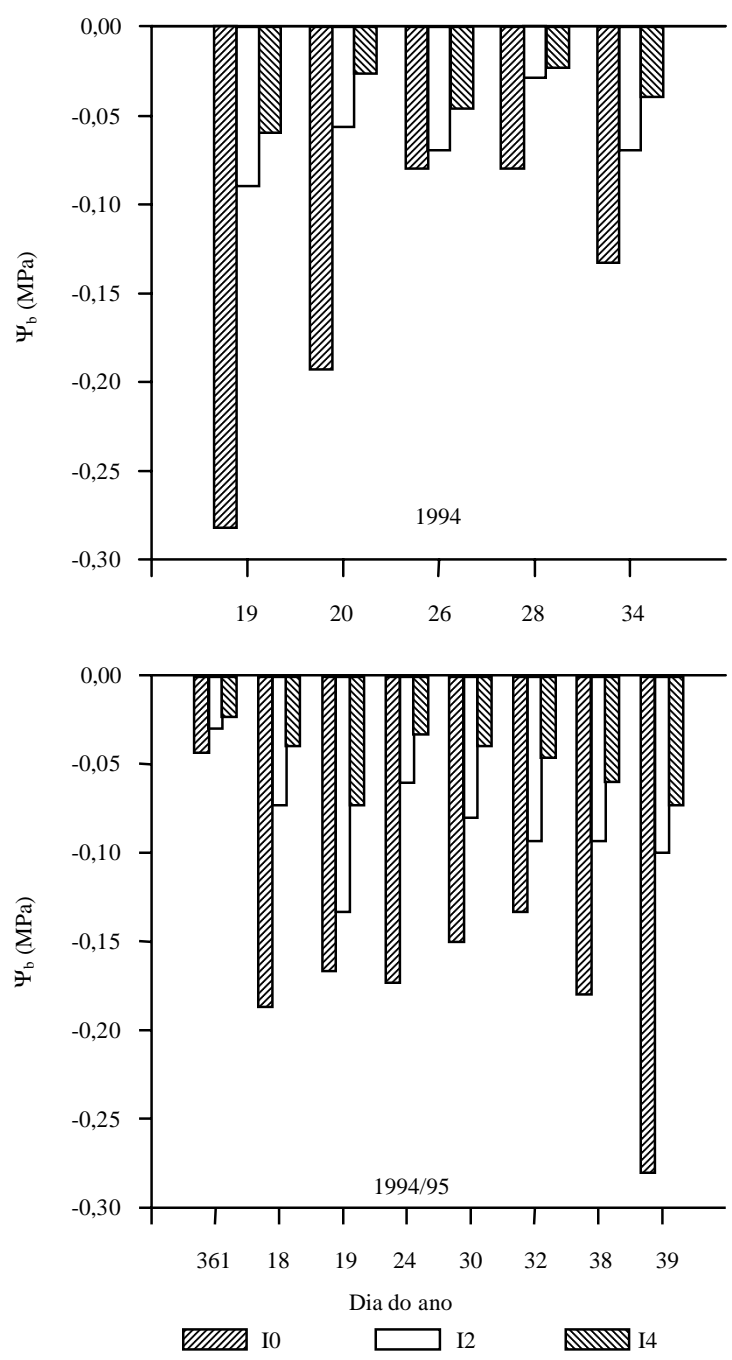

FIG. 4. Potencial de base $\left(\psi_{\mathrm{b}}\right)$ em milho submetido aos tratamentos: sem irrigação (I0); dose de rega igual a $75 \%$ de I4 (I2) e irrigação para manter o solo próximo à capacidade de campo (I4). Eldorado do Sul, RS.

Pesq. agropec. bras., Brasília, v.35, n.8, p.1531-1540, ago. 2000 
dos semelhantes foram obtidos por Tardieu et al. (1990), em que o potencial de base, em milho, permaneceu elevado apesar de o potencial da água no solo ser baixo, atribuindo tal comportamento a um fluxo lento de água das camadas não exploradas pelas raízes para o sistema radicular. Segundo Katerji \& Hallaire (1984), o potencial de base depende do estado hídrico do solo, da densidade e da profundidade do sistema radicular. Por vezes, as pequenas diferenças observadas entre os tratamentos podem também ser explicadas, em parte, pela formação de orvalho nas plantas, cuja água seria absorvida pelas folhas, conforme sugerido por Ceulemans et al. (1988). Os mesmos autores observaram que o potencial da água na folha medido entre as $8 \mathrm{~h}$ e $16 \mathrm{~h}$ é melhor indicador de déficit hídrico do que o potencial de base, em estudos de trocas gasosas em milho. Os dados apresentados por Ceulemans et al. (1988) sugerem que o potencial de base, em milho, não representa o verdadeiro equilíbrio com a umidade do solo. Para esses autores, a idéia da correspondência entre o potencial de base e o potencial matricial médio do solo não se aplica quando a umidade do solo for muito heterogênea. Outra causa para as pequenas diferenças poderia ser o fato de que, no momento das medições (6h) em virtude da luz já existente, principalmente azul, os estômatos estariam parcialmente abertos. Desta forma, ao penetrar na folha, o orvalho alteraria o potencial de base, não ocorrendo, então, a condição de equilíbrio entre a planta e o solo. Apesar das controvérsias existentes na literatura o potencial de base é apresentado, de modo geral, como um dos melhores indicadores de déficit hídrico em milho (Dwyer \& Stewart, 1984; Tardieu et al., 1990) e em Prosopis glandulosa (Ansley et al., 1992).

Pelos resultados obtidos, o milho demonstra ser uma planta de comportamento aniso-hídrico, ou seja, não mantém o potencial da água alto nos tecidos nas horas mais quentes do dia (Figs. 1 e 2). Neste caso, segundo Katerji et al. (1988), o potencial mínimo da água na folha (no início da tarde) é um bom critério indicador de déficit hídrico, ao contrário do que ocorre com plantas iso-hídricas. O abaixamento do potencial da água nas horas mais quentes do dia reflete, na verdade, o efeito da deficiência hídrica no solo. Nestas condições, se a transpiração for elevada e maior do que a absorção de água pelo sistema radicular, desde as primeiras horas do dia, o potencial da água em plantas não irrigadas diminui, permanecendo em patamares baixos até o entardecer.

Na Fig. 5 está representada uma relação entre o potencial da água no solo e o potencial mínimo da água na folha (às $15 \mathrm{~h}$ ), podendo-se verificar que ela é consistente. Isto mostra a dependência do potencial da água na folha à disponibilidade de água no solo. Embora haja elevada associação entre as variáveis, observa-se certa dispersão de pontos, a qual pode ser devida às resistências implicadas no processo de absorção e no fluxo de água, principalmente com relação às resistências do solo e da raiz, as quais aumentam em condições de baixa disponibilidade hídrica. A mesma tendência de associação tem

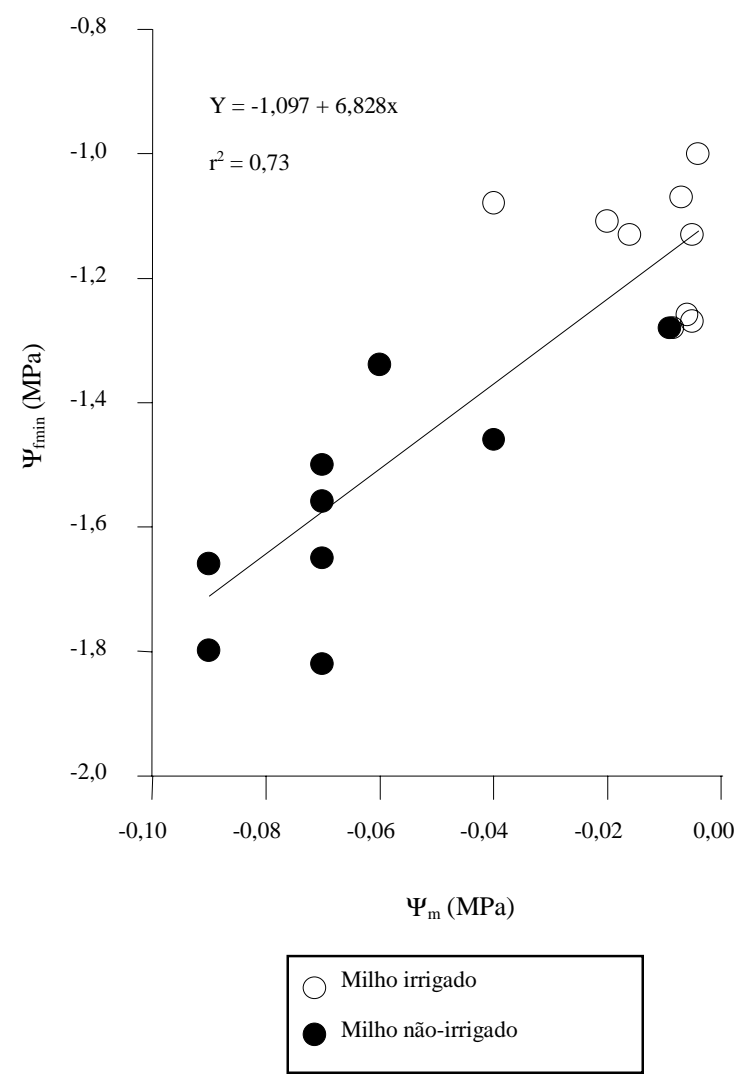

FIG. 5. Relação entre o potencial matricial da água no solo $\left(\Psi_{\mathrm{m}}\right)$ e o potencial mínimo de água na folha $\left(\psi_{\text {fmin }}\right)$ em milho irrigado e não-irrigado. Eldorado do Sul, RS. 
sido observada por vários autores, não só com milho, mas também com outras culturas (Grimes \& Yamada, 1982; Bittman \& Simpson, 1989; Tardieu et al., 1990). Para Ceulemans et al. (1988), a queda do potencial da água na folha, em milho, esteve associada às condições atmosféricas (temperatura e déficit de pressão de vapor) e ao déficit de água no solo.

\section{CONCLUSÕES}

1. O potencial da água na folha ao entardecer, $\log g$ após o fechamento estomático, está relacionado à intensidade do déficit hídrico (expressa pelo potencial foliar mínimo), e pode ser utilizado como indicador de déficit hídrico em milho.

2. O potencial mínimo da água na folha tem relação linear com o potencial matricial da água no solo, a $45 \mathrm{~cm}$ de profundidade.

3. Em condições de campo, a cultura do milho apresenta comportamento aniso-hídrico, cujo potencial da água na folha ocorre entre as $12 \mathrm{~h}$ e $15 \mathrm{~h}$ (horário local).

\section{REFERÊNCIAS}

ANSLEY, R.J.; JACOBY, P.W.; MEADORS, C.H.; LAWRENCE, B.K. Soil and leaf water relations of differentially moisture stressed honey mesquite (Prosopis glandulosa Torr). Journal of Arid Environments, London, v.22, n.1, p.147-159, 1992.

BENNETT, J.M. Problems associated with measuring plant water status. HortScience, Alexandria, v.25, n.12, p.1551-1554, 1990.

BERGAMASCHI, H.; GUADAGNIN, M.R. Agroclima da estação experimental agronômica/UFRGS. Porto Alegre : UFRGS-Faculdade de Agronomia, 1990. 60p.

BITTMAN, S.; SIMPSON, G.M. Drought effects on water relations of three cultivated grasses. Crop Science, Madison, v.9, n.4, p.992-999, 1989.

BOYER, J.S. Leaf enlargement and metabolic rates in corn, soybean and sunflower at various leaf water potentials. Plant Physiology, Rockville, v.46, n.2, p.233-235, 1970.
BOYER, J.S. Leaf water potentials measured with a pressure chamber. Plant Physiology, Rockville, v.42, n.1, p.213-217, 1967.

CEULEMANS, R.; IMPENS, I.; LAKER, M.C.; ASSCHE, F.M.G. van; MOTRAN, R. Net $\mathrm{CO}_{2}$ exchange rate as a sensitive indicator of plant water status in corn (Zea mays L.). Canadian Journal of Plant Science, Ottawa, v.68, n.3, p.597-606, 1988.

CROMBIE, D.S.; TIPPETT, J.T.; HILL, T.C. Dawn water potential and root depth of trees and under storey species in South-Western Australia. Australian Journal of Botany, Collingwood, v.36, p.621-631, 1988.

CUNHA, G.R. da. Evapotranspiração e função de resposta à disponibilidade hídrica em alfafa. Porto Alegre : UFRGS, 1991. 198p. Tese de Doutorado.

DWYER, L.M.; STEWART, D.W. Indicators of water stress in corn (Zea mays L.). Canadian Journal of Plant Science, Ottawa, v.64, n.3, p.537-546, 1984.

EHRLER, W.L.; IDSO, S.B; JACKSON, R.D.; REGINATO, R.J. Diurnal changes in plant water potential and canopy temperature of wheat as affected by drought. Agronomy Journal, Madison, v.70, n.6, p.999-1004, 1978.

ELFVING, D.C.; KAUFMAN, M.R.; HALL, A.E. Interpreting leaf water potential measurements with a model of the soil-plant-atmosphere continuum. Physiologia Plantarum, Copenhagen, v.27, p.161168, 1972.

FAIZ, S.M.A.; WEATHERLY, P.E. Further investigations into the location and magnitude of the hydraulic resistances in the soil-plant system. New Phytologist, Cambridge, Grã-Bretanha, v.81, n.1, p.19-28, 1978.

GRIMES, D.W.; YAMADA, H. Relation of cotton growth and yield to minimum leaf water potential. Crop Science, Madison, v.22, n.1, p.134-139, 1982.

HANKS, R.J.; KELLER, J.; RASMUSSEN. V.P.; WILSON, G.D. Line source sprinkler for continuous variable irrigation-crop production studies. Soil Science Society of America. Journal, Madison, v.40, n.3, p.426-429, 1976.

HANKS, R.J.; SISSON, D.V.; HURST, R.L.; HUBARD, K.G. Statistical analysis of results from irrigation experiments using the line-source sprinkler system. Soil Science Society of America. Journal, Madison, v.44, n.4, p.886-888, 1980.

Pesq. agropec. bras., Brasília, v.35, n.8, p.1531-1540, ago. 2000 
HSIAO, T.C. Plant responses to water stress. Annual Review of Plant Physiology, Palo Alto, v.24, p.519570, 1973.

KATERJI, N.; HALLAIRE, M. Les grandeurs de référence utilisables dans l'étude de l'alimentation en eau des cultures. Agronomie, Paris, v. 4, n.10, p.999-1008, 1984.

KATERJI, N.; ITIER, B.; FERREIRA, I. Etude de quelques critères indicateurs de l'état hydrique d'une culture de tomate en région semi-aride. Agronomie, Paris, v.8, n.5, p.425-433, 1988.

MORISON, J.I.L.; GIFFORD, R.M. Plant growth and water use with limited water supply in high $\mathrm{CO}_{2}$ concentrations. II. Plant dry weight, partitioning and water use efficiency. Australian Journal of Plant Physiology, Collingwood, v.11, p.375-384, 1984.

MORIZET, J.; TOGOLA, D. Effect et arrière-effect de la sécheresse sur la croissance de plusieurs génotypes de maïs. In: CONFERENCE INTERNATIONALE DES IRRIGATIONS ET DU DRAINAGE, 1984, Versailles. Les bésoins en eau des cultures. Paris : INRA, 1984. p.351-360.

PANDEY, R.K.; HERRERA, W.A.; PENDLETON, J.W. Drought response of grain legumes under irrigation gradient. II. Plant water status and canopy temperature. Agronomy Journal, Madison, v.76, n.4, p.553-557, 1984.

PREMACHANDRA, G.S.; SANEOKA, H.; FUJITA, K.; OGATA, S. Osmotic adjustment and stomatal response to water deficits in maize. Journal of Experimental Botany, Oxford, v.43, p.1451-1456, 1992.

REICH, P.B.; HINCKLEY, T.M. Influence of pre-dawn water potential and soil-to-leaf hydraulic conductance on maximum daily leaf diffusive conductance in two oak species. Ecology, New York, v.3, p.719-726, 1989.

REICOSKY, D.C.; RITCHIE, J.T. Relative importance of soil resistance and plant resistance in root water absorption. Soil Science Society of America. Journal, Madison, v.40, n.2, p.293-297, 1976.

ROJAS, C.A.L. Alterações físico-hídricas de um podzólico em função do manuseio do solo. Porto Alegre : UFRGS, 1998. 76p. Dissertação de Mestrado.

SHARP, R.E.; DAVIES, W.J. Solute regulation and growth by roots and shoots of water stressed maize plants. Planta, Berlin, v.147, n.1, p.43-49, 1979.

SIONIT, N.; KRAMER, P.J. Water potential and stomatal resistance of sunflower and soybean subjected to water stress during various growth stages. Plant Physiology, Rockville, v.58, n.4, p.537-540, 1976.

SLATYER, R.O. Plant-water relationships. New York : Academic, 1967. 366p.

TANGUILIG, V.C.; YAMBAO, E.B.; O’TOOLE, J.C.; DE DATTA, S.K. Water stress effects on leaf elongation, leaf water potential, transpiration, and nutrient uptake of rice, maize, and soybean. Plant and Soil, Dordrecht, v.103, n.1, p.155-168, 1987.

TARDIEU, F.; KATERJI, N.; BETHENOD, O. Relations entre l'état hydrique du sol, le potentiel de base et d'autres indicateurs de la contrainte hydrique chez le maïs. Agronomie, Paris, v.10, n.8, p.617-626, 1990.

WESTGATE, M.E. Water status and development of the maize endosperm and embryo during drought. Crop Science, Madison, v.34, n.1, p.76-83, 1994. 